\title{
Vertebral Compression Fracture after removing Implants in A Patient undergoing Posterior Lumbar Interbody Fusion: A Case Report and Literature Review
}

\author{
Ji Won Nam, Jong Min Lee, In Uk Lyo, Soon Chan Kwon, Hong Bo Sim \\ Department of Neurological Surgery, Ulsan University Hospital, University of Ulsan College of Medicine, Ulsan, Republic of Korea
}

Corresponding author: Jong Min Lee, MD Department of Neurosurgery, Ulsan University Hospital, University of Ulsan College of Medicine, 877 Bangeojinsunhwando-ro, Dong-gu, Ulsan 44033, Republic of Korea

Tel: $+82-52-250-7139$

Fax: $+82-52-250-7138$

E-mail: gogmgek@naver.com

Received: February 01, 2021

Revised: February 24, 2021

Accepted: March 02, 2021
Posterior lumbar interbody fusion (PLF) is a well-known method for treating spinal diseases in which adjacent segment disease (ASD) is a representative complication. To correct ASD, posterior fusion is extended to the upper or lower segment, and the previous instruments are removed. However, there have been a few reports of complications after instrument removal. Here, we report on a case of compression fracture that occurred without history of trauma after ASD revision and instrument removal surgery. A 68-year-old woman underwent PLF at L3-L5 and was hospitalized for treatment of ASD. She underwent oblique lumbar interbody fusion at $\mathrm{L} 2-\mathrm{L} 3$ after removal of the previous screws. One month later, she visited the hospital with sudden lower back pain. Plain radiography revealed a compression fracture of L4. The patient's pain was relieved after conservative treatment. Our findings show that instrument removal during revision operations should be performed carefully because it can lead to compression fracture without history of trauma.

Key Words: Fractures, Compression, Pedicle Screws, Spinal Fusion

\section{INTRODUCTION}

Interbody fusion using pedicle screws, such as posterior lumbar interbody fusion (PLIF), oblique lumbar interbody fusion (OLIF), direct lateral interbody fusion (DLIF), and transforaminal lumbar interbody fusion (TLIF), is a widely used method in the treatment of spinal diseases $^{1,3,5}$. However, complications associated with this procedure include adjacent segment disease (ASD), pseudoarthrosis, screw loosening, and infection ${ }^{2}$. In cases of ASD, it is common to extend spine fusion toward the upper or lower segment; however, there have been few reports of complications caused by the removal of previously inserted pedicle screws. Here, we present a case of vertebral compression fracture after removal of pedicle screws in a patient without history of trauma.

\section{CASE REPORT}

A 68-year-old woman visited the outpatient clinic with pain in the anterior portion of her right thigh. She had a history of PLIF at L3-L5 due to degenerative spinal stenosis in September 2011. She also complained of severe neurogenic intermittent claudication (NIC) and was unable to walk more than $50 \mathrm{~m}$. Upon physical examination, pain was noted along the right $\mathrm{L} 2$ sensory dermatome, and the femoral nerve stretch test result was positive. Due to adjacent segment degeneration from the previous L3-L5 PLIF, a reduction in disc height, presence of a radiolucent lesion with vacuum inside the disc space, as well as sclerotic changes in both end plates at L2L3 were observed on plain radiograph images (Figure 1A, B). Computed tomography revealed a solid bony fusion between $\mathrm{L} 3$ and L5 without evidence of pedicle screw loosening (Figure 2). Magnetic resonance imaging revealed severe foraminal stenosis on the right side (Figure 3). A bone mineral density (BMD) reading showed a value of -1.5, indicating osteopenia (Figure 4A, B). To treat the right
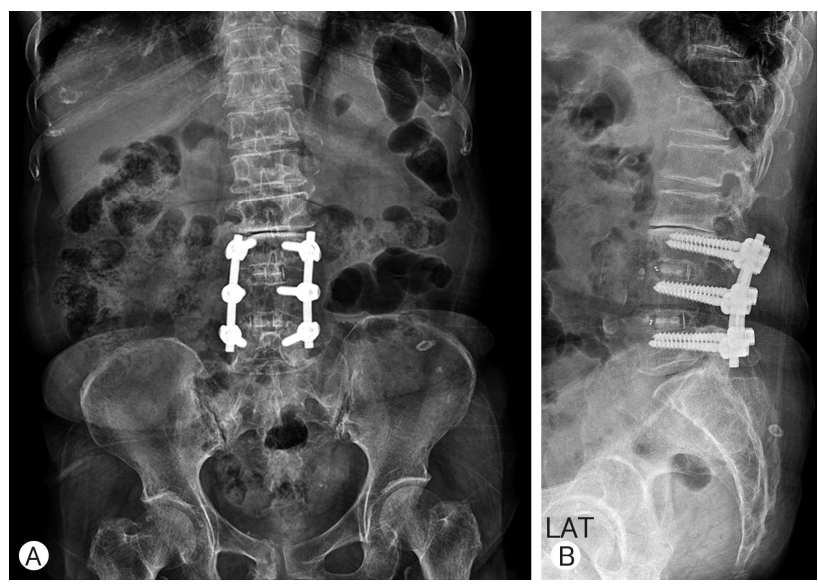

Figure 1. Preoperative anteroposterior (A) and lateral (B) radiographs showing previous L3-L5 interbody fusion state, reduced disc height, presence of a radiolucent lesion with vacuum inside the disc space, and sclerotic changes in both end plates at L2-L3. 
leg pain caused by the adjacent segment degeneration in L2-L3 after the patient's previous PLIF, analgesics were administered and a selective root block was performed. However, as her symptoms continued to worsen, revision surgery was performed in February 2020. Since a solid bony fusion had previously formed between $\mathrm{L} 3$ and $\mathrm{L} 5$, the oblique lumbar interbody fusion of L2-L3 was performed after removing the previous instruments (Figure 5A, B). She showed good clinical progress and was discharged on postoperative day 14. At 2 months post-discharge, she visited the outpatient clinic complaining of sudden low back pain. She had no history of trauma, but a compression fracture of $L 4$ was observed on plain radiography (Figure 6-A, B). On the laboratory test, the leukocyte count was 5,850 and C-reactive protein (CRP) was 0.26 , and there were no

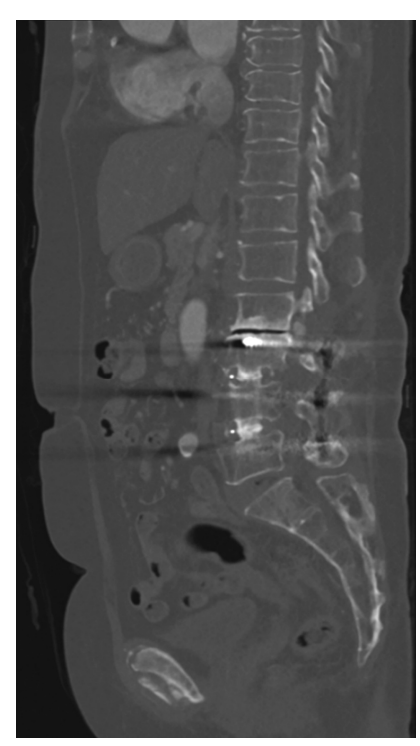

Figure 2. Preoperative lumbar spine computed tomography sagittal image showing solid bony fusion between $L 3$ and $L 5$ without evidence of pedicle screw loosening.

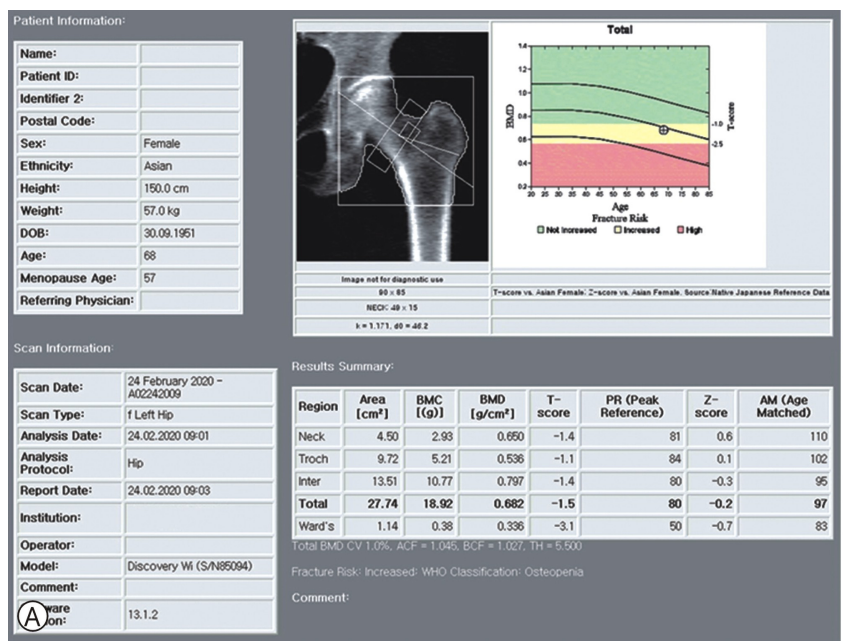

other specific findings. After brace application and teriparatide injection, her back pain was relieved, and she was discharged with good clinical progress.

\section{DISCUSSION}

Although spinal fusion is a widely used treatment for various spinal diseases, there are a few reports of complications associated with the removal of fusion implants. For cases of posterolateral fusion, there are several reports of vertebral fractures occurring after screw removal ${ }^{8,9,15}$. In these cases, it was reported that the bending moment exerted on the pedicle by the cantilever effect may lead to pedicle

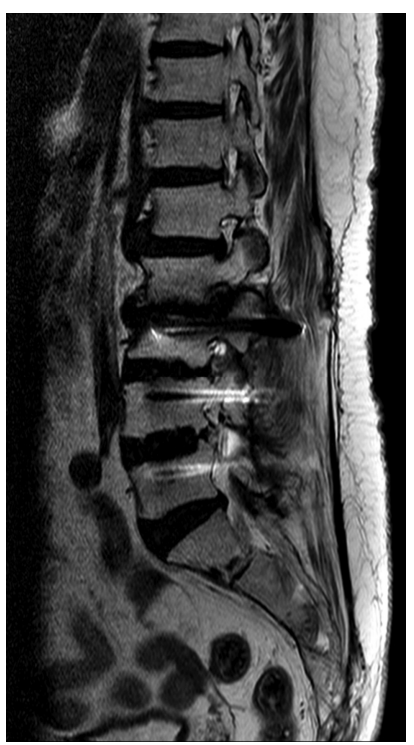

Figure 3. Preoperative magnetic resonance image showing severe foraminal stenosis on the right side of L2-L3.

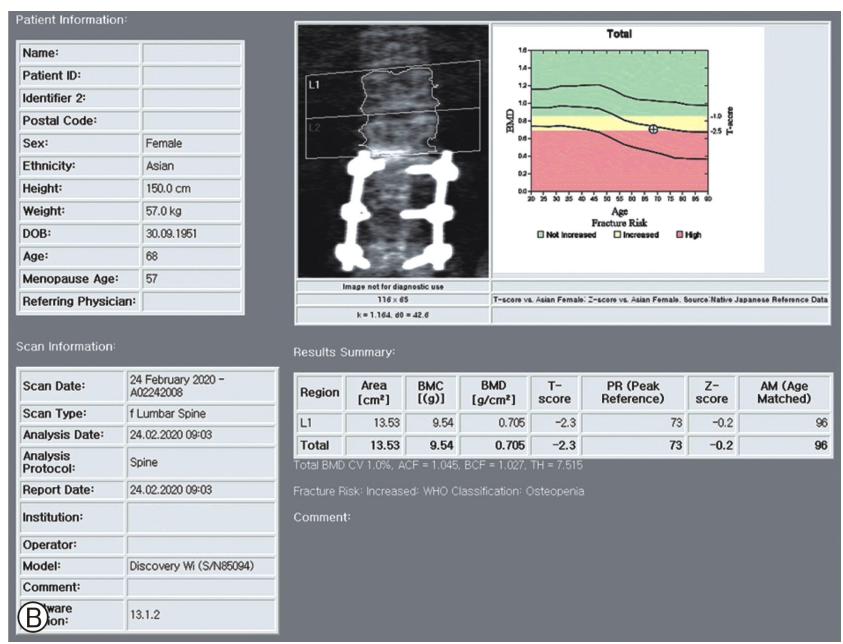

Figure 4. Preoperative bone densitometry taken from hip (A) and lumbar spine (B) showed a value of -1.5 indicating osteopenia. 

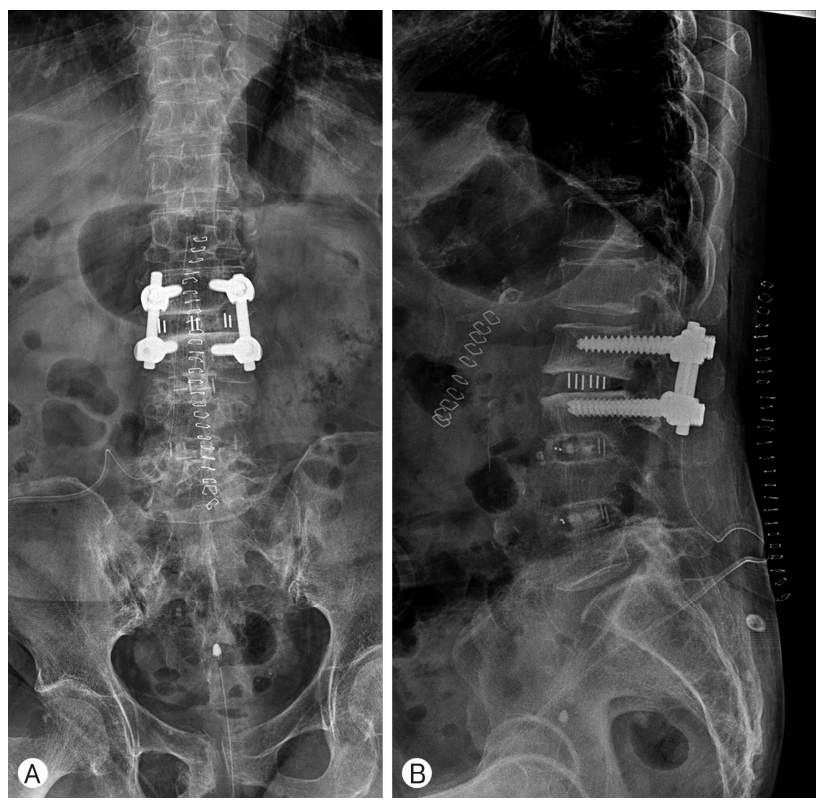

Figure 5. Postoperative anteroposterior (A) and lateral (B) radiographs showing that solid bony fusion had formed between L3 and L5, the removal of previous instruments, and an oblique lumbar interbody fusion state at L2-L3.
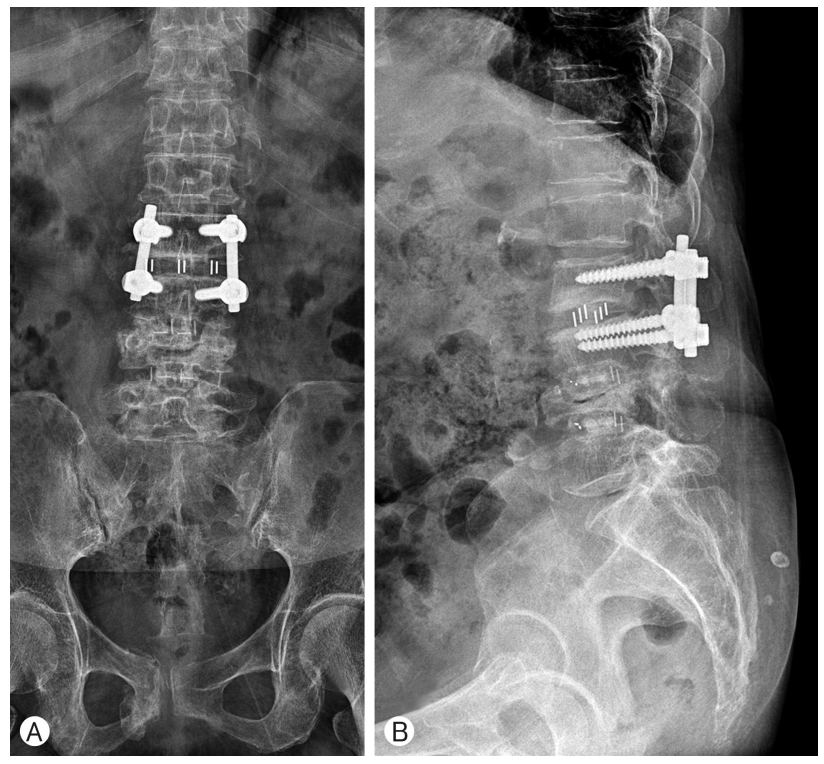

Figure 6. Anteroposterior (A) and lateral (B) radiographs taken at 1 month after surgery showing a compression fracture of $L 4$.

and vertebral body fracture $4,7,9,10,14$. However, there have been few reports of compression fractures occuring within the fused vertebral body through interbody fusion ${ }^{11}$. The vertebral body is thought to withstand the cantilever effect and axial force better in cases of interbody fusion than in cases of posterolateral fusion because it is the fused anterior column. However, as seen in our patient, fractures can also occur inside the fused vertebral mass, suggesting that remo- val of fusion implants may negatively affect patients, even those with interbody fusion. To the best of our knowledge, this is the first case report to describe a vertebral compression fracture in the center of a fused segment through an interbody fusion operation. Mataki et al. suggested that removing pedicle screws while the posterior element is removed due to PLF increases stress on the vertebral body $^{11}$. It is also possible that stress shielding-related osteopenia occurred in the patient ${ }^{6,12,13}$.

This case has several implications for the treatment of patients with ASD. First, in most cases of ASD, such as in this patient, the bone mineral density of the fused vertebral body cannot be directly measured due to fusion from previous instrumentation, despite the possibility of stress shielding-related osteopenia. Vertebral body osteopenia may occur as a result of instrumented spinal fusion. In an experimental environment, osteopenia worsened in proportion to the time at which stress shielding occurred ${ }^{6}$. This suggests that local bone quality, especially in cases of a fused vertebral mass, cannot be guaranteed in patients undergoing long instrumentation times, even in those with healthy bone mineral density. Furthermore, even if computed tomography can indirectly check bone quality, accurate determination is difficult when screws are inserted into the vertebral body due to metallic artifacts. Kim et al. reported that evidence of decreased bone quality on plain lumbar radiographs includes an overall increase in radiolucency of the vertebral body, loss of trabecular bone, thinning of the cortex, and the presence of well-demarcated cortical rim?. However, given the fact that many factors can affect the image quality of plain radiographs, the question remains as to whether these findings can be used as a basis for prospective judgment.

\section{CONCLUSION}

Vertebral compression fractures in the center of a fused segment, especially in the middle of the fused body in cases of interbody fusion, after removing fusion implants are possible but occur rarely. In patients with osteopenia or long periods of instrumentation, removal of fusion instruments should be seriously considered, and sufficient explanation should be given to the patient.

\section{REFERENCES}

1. Boos N, Webb J: Pedicle screw fixation in spinal disorders: A European view. Eur Spine J 6:2-18, 1997

2. Cho KJ, Suk SI, Park SR, Kim JH, Kim SS, Choi WK, et al.: Complications in posterior fusion and instrumentation for degenerative lumbar scoliosis. Spine 32:2232-2237, 2007

3. Crawford MJ, Esses SI: Indications for pedicle fixation. Results of NASS/SRS faculty questionnaire. North American Spine Society and Scoliosis Research Society. Spine 19:2584-2589, 1994

4. Deckey JE, Bradford DS: Loss of sagittal plane correction after removal of spinal implants. Spine 25:2453-2460, 2000

5. Foley KT, Holly LT, Schwender JD: Minimally invasive lumbar 
fusion. Spine 28:S26-S35, 2003

6. Globus RK, Bikle DD, Morey-Holten E: Effects of simulated weightlessness on bone mineral metabolism. Endocrinology 114: 2264-2270, 1984

7. Ha KY, Kim YH: Bilateral pedicle stress fracture after instrumented posterolateral lumbar fusion: A case report. Spine 28: E158-E160, 2003

8. Ha KY, Kwon SE, Kim KW, Oh IS, Lee YM: Vertebral compression fracture in the middle of fused segments without a history of injury: A case report. Spine 35:E137-E139, 2010

9. Kim SK, Chung JY, Seo HY, Lee WG: Vertebral compression fracture within a solid fusion mass without trauma after removal of pedicle screws. Spine 16:e219-e223, 2016

10. Macdessi SJ, Leong AK, Bentivoglio JE: Pedicle fracture after instrumented posterolateral lumbar fusion: A case report. Spine 26:580-582, 2001
11. Mataki K, Fukushima M, Kaneoka K, Ikeda K, Kumagai H, Nagashima K, et al.: Vertebral fracture after removing pedicle screws used for posterior lumbar interbody fusion: A case report. J Clin Neurosci 57:182-184, 2018

12. McAfee P: Device-related osteoporosis with spinal instrumentation. 1989 Volvo Award in Basic Science. Spine 14:919-926, 1989

13. Myers MA, Casciani T, Whitbeck Jr GM, Puzas EJ: Vertebral body osteopenia associated with posterolateral spine fusion in humans. Spine 21:2368-2371, 1996

14. Rathjen K, Wood M, McClung A, Vest Z: Clinical and radiographic results after implant removal in idiopathic scoliosis. Spine 32:2184-2188, 2007

15. Waelchli B, Min K, Cathrein P, Boos N: Vertebral body compression fracture after removal of pedicle screws: A report of two cases. Eur Spine J 11:504-506, 2002. 